\title{
The efficiency of different simulation-based design methods in improving building performance
}

\author{
A.-T. Nguyen ${ }^{1} \&$ S. Reiter ${ }^{2}$ \\ ${ }^{1}$ Faculty of Architecture, Danang University of Science and Technology, \\ Viet Nam \\ ${ }^{2}$ LEMA, Faculty of Applied Sciences, University of Liege, Belgium
}

\begin{abstract}
This paper presents a pioneering effort to define the efficiency of the most common simulation-based design methods, namely the parametric simulation method (PSM) and the simulation-based optimization method (SOM), in improving two building performance indicators: thermal comfort and energy consumption. Three case-study houses were selected and their indoor conditions were continuously monitored during one summer month in 2012. Computer EnergyPlus models of these houses were established and then carefully calibrated by the monitoring data to improve the reliability of the numerical methods. Thermal performances of these houses during a year were simulated, then improved by the PSM and finally optimized by the SOM. By comparing the results of these two simulation-based design methods, this research found that both the PSM and SOM were very effective in improving these building performance indicators. This study found that the SOM is almost two times more efficient than the PSM in improving thermal comfort in naturally ventilated (NV) houses and life cycle cost in air-conditioned (AC) houses. On average, the discomfort period in NV houses could be reduced by $44.9 \%$ by the PSM while as high as $86.1 \%$ by the SOM, compared with the reference cases. The life cycle cost of AC houses had smaller reductions with $6.2 \%$ and $14.6 \%$ cut-off by the PSM and SOM, respectively. The results of this work give a strong and explicit insight of the actual efficiency of each design method. This work also shows great advantages of applications of advanced numerical approaches in the design of high performance buildings.
\end{abstract}

Keywords: simulation efficiency, simulation-based method, building optimization, numerical calibration. 


\section{Introduction and research objective}

Numerical simulation has become more and more common in building research, design and consultancies. However, it is still unclear whether a simulation-based design method can help designers to satisfy a certain threshold of building performance. It is important to know the capability of simulation-based optimization methods in improving a design objective such as indoor environment quality or building energy consumption. This allows designers to choose an appropriate method among a number of available approaches that can satisfy their time budget, resources and design objectives.

To accurately give an answer to this question, this research developed a comprehensive framework through which the efficiency of two major simulationbased design methods, i.e. the parametric simulation method (PSM) and simulation-based optimization method (SOM), in improving building performance could be accurately quantified. The results presented in this paper are part of a larger research project aimed to optimize indoor thermal comfort and energy consumption in residential buildings. We only performed the survey on residential buildings in hot humid climates. Nevertheless, the procedure applied in this paper can be used for the same purposes in other climatic regions and other building types.

\section{Methodology}

The reliable results of this study were based on several stringent evaluation criteria:

The reference performance of case-study buildings must be carefully defined.

The design targets to be improved, the building styles, the climates to be investigated must be abundant to avoid exceptional bias.

The authors first developed the reference performance of a number of residential buildings by selecting 3 case-study houses, including a row house, a detached house and an apartment (see Fig. 1). These case-study houses are located in Danang, Vietnam where the climate is hot and humid all year round. They are typical in style and size in the region and were designed by CAD-based techniques without using building performance simulation tools.

Thermal conditions in representative zones of these houses were continuously monitored during one summer month ( $1^{\text {st }}$ to $31^{\text {st }}$ May 2012) by using HOBO data loggers (see locations in Fig. 1). Hourly outdoor conditions during the monitoring period were booked and obtained from a national meteorological station in Danang city. Computer thermal models of these houses were established using the EnergyPlus simulation program. The monitoring data were then used to calibrate these models using the method of Nguyen and Reiter [1].

The case-study houses were assumed being located in 3 climatic regions of Vietnam, including Hanoi ( $21^{\circ}$ North), Danang ( $16^{\circ}$ North) and Hochiminh city $\left(10^{\circ}\right.$ North). 1-year EnergyPlus simulation results of calibrated computer thermal models were considered as the reference performances of the case-study houses. 
Thermal performances of the case-study houses were improved by the PSM and the SOM. The efficiency of the PSM and SOM can, therefore, be derived by comparing the improved performances with the reference counterparts.

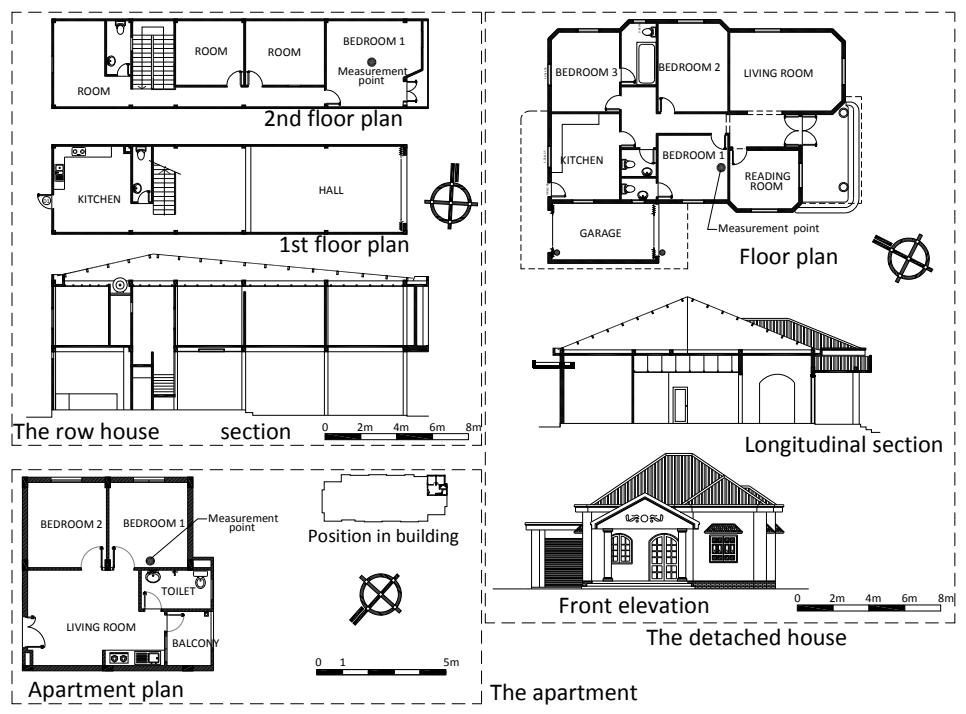

Figure 1: Three case-study houses and locations of measuring equipment.

\section{Calibration of computer thermal models}

The calibration of a numerical building model by measured data has been recognized as an important factor in substantiating how well the model fits the data from the actual building. In this case, to analyze the overall thermal performance of the case-study houses, the simulated indoor temperature during the baseline period was compared against the average measured temperature to judge how successful the simulation was.

An Airflow network model was coupled with the thermal-energy module in EnergyPlus to accurately reproduce the thermal-airflow phenomena in naturally ventilated case-study houses. To provide inputs to the airflow network model, the wind pressure coefficients (WPC) on building facades corresponding to 12 major wind directions were calculated by CFD simulations on building models. Fig. 2 demonstrates an example (among 36 results of the 3 houses) of the WPC distribution on the apartment building given by a CFD simulation on Phoenics code.

The agreement between measured and simulated air temperature of the houses was assessed by two statistical indicators, namely Normalized Mean Bias Error (NMBE) and Coefficient of Variation of Root Square Mean Error CV (RMSE), defined as follows: 


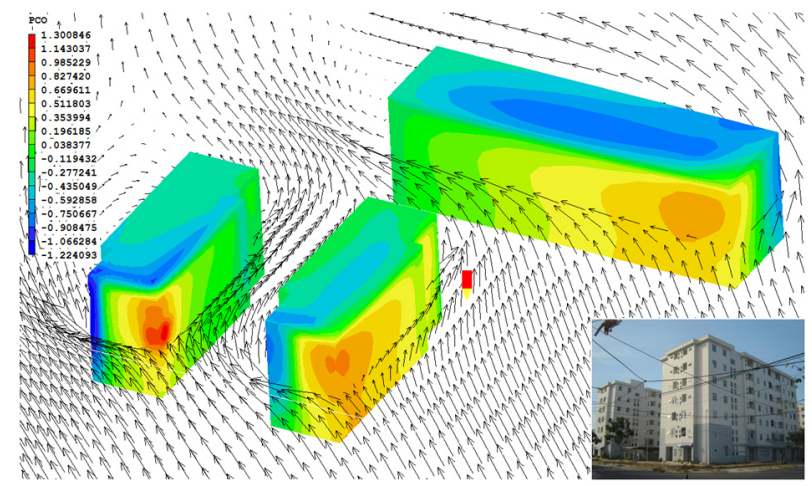

Figure 2: Simulated WPC surface contours of the wind from the South - the apartment is on the 4 th floor of the building on the left.

$$
\begin{gathered}
\operatorname{NMBE}(\%)=\frac{\sum_{i=1}^{n}\left(t_{i p}-t_{i m}\right)}{n-1} \frac{1}{t_{m}} \\
C V(R M S E)(\%)=\sqrt{\frac{\sum_{i=1}^{n}\left(t_{i p}-t_{i m}\right)^{2}}{n-1}} \frac{1}{t_{m}} 100 \%
\end{gathered}
$$

where $t_{i p}$ is the $i_{t h}$ predicted temperature by the simulation; $t_{i m}$ is the $i_{t h}$ measured temperature; $t_{m}$ is the arithmetic mean of the sample of $\mathrm{n}$ measurements and $n$ is the number of data points (temperature) in the measurement.

These indicators were iteratively improved by adjusting inputs of the EnergyPlus models until NMBE and CV(RMSE) reach convergence (no or very small change). Ideally, both NMBE and CV(RMSE) should be zero, indicating a perfect "goodness-of-fit". Fig. 3 shows the improvement process of NMBE and CV(RMSE) and the "goodness-of-fit" between simulated and measured temperature in the calibration of the row house.

\section{Reference performance of the case-study houses}

After the calibration was finished, calibrated models were run for 1-year period using TMY weather files of 3 locations. The case-study houses were assumed that they could be operated in two separated operation modes: naturally ventilated (NV) and air conditioned (AC). In the NV mode, all windows are always opened to allow natural ventilation (except that they are closed during November, December, January and February in Hanoi). All doors are normally opened during day time and closed during nighttime. In the AC mode, each thermal zone was equipped with a Packed Terminal Air Conditioner (PTAC). Each PTAC consists of an electric heating coil, a single-speed cooling coil, a 'draw through' fan, an outdoor air mixer, a thermostat control and a temperature sensor. Total energy consumption of the houses is the sum of electricity consumed by the lighting system, equipment and the PTACs. 

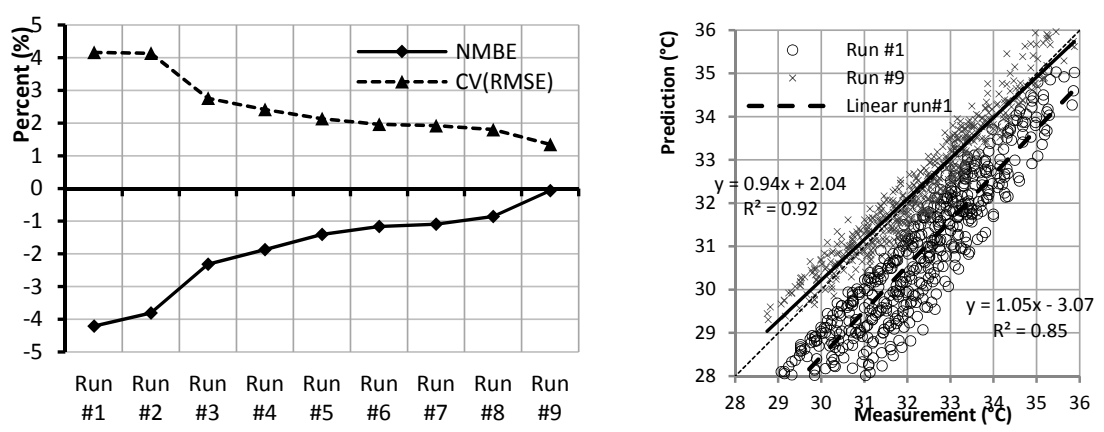

Figure 3: Tuning progress with input modifications and linear regression analysis of run \#1 vs. run $\# 9$ - row house.

Two building performance indicators were used in this study. The first is the Total Discomfort Hours (TDH) applied to the NV houses which are defined by cumulative uncomfortable hours in a year (average of all thermal zones) with respect to the adaptive thermal comfort criterion developed in [2]:

$$
T_{\text {comf }}=0.341 T_{\text {out }}+18.83
$$

where $T_{\text {out }}$ is monthly mean outdoor temperature; $T_{\text {comf }}$ is predicted comfort temperature.

The second indicator is the Life Cycle Cost (LCC) applied to the AC houses which is defined by the sum of initial construction cost, energy cost and other maintenance cost during 50 years (in US dollar). References [3] and [4] describe these indicators in detail. A low TDH or LCC is obviously preferred.

The TDHs and the LCCs of the case-study houses under the 3 climatic regions are shown in Fig. 4 and Fig. 5. These data give an estimate of how well the casestudy houses are. As can be seen, the TDHs of all reference cases were not acceptable and were classified as "POOR" (TDH $>10 \%)$ [5].

\section{Efficiency of the PSM}

This section looks for climate responsive design strategies and building control with an aim of improving the reference TDHs and LCCs. Following a trial-anderror procedure of numerical simulations, the parametric simulation method (PSM) allows users to explore the effect of each design variable on the simulation outputs; hence it is able to provide improved solutions to the problem.

In this study, a number of common strategies were subsequently applied to improve the TDHs and LCCs as listed below:

- Changing the structure of external walls;

- Installing thermal insulation beneath the roof;

- $\quad$ Changing the color of external walls (changing solar absorptance);

- Controlling openings according to different ventilation schemes; 
- Other strategies, i.e. adjusting: window overhang, size of glazing area, glazing type, air infiltration rate, and internal thermal mass.

In each house after exploring all these solutions separately, a combined solution which was constituted from all positive solutions was applied to obtain the maximum improvement. Fig. 4 and 5 compares the reference TDHs and LCCs with the improved counterparts given by the PSM.

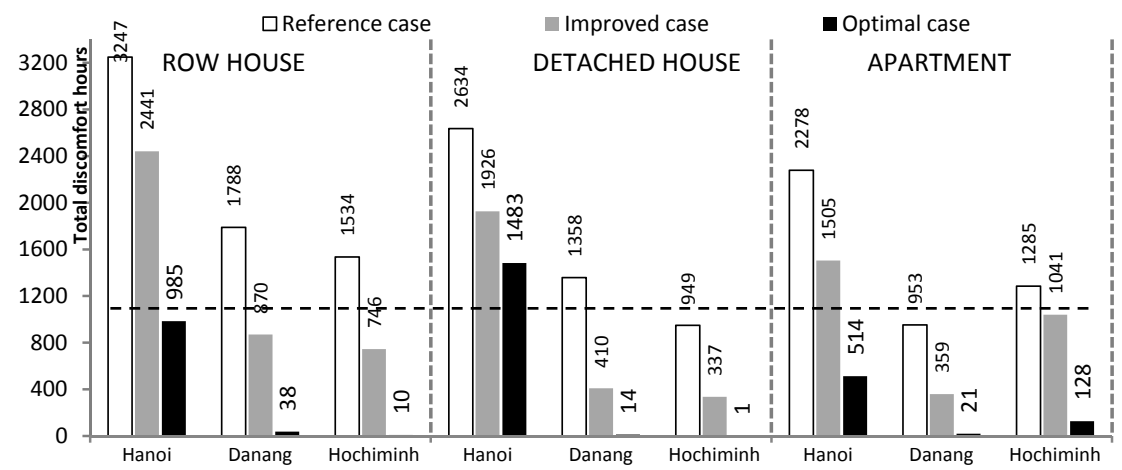

Figure 4: Comparison of the TDHs in the NV houses. The horizontal line indicates the acceptable threshold of the TDH.

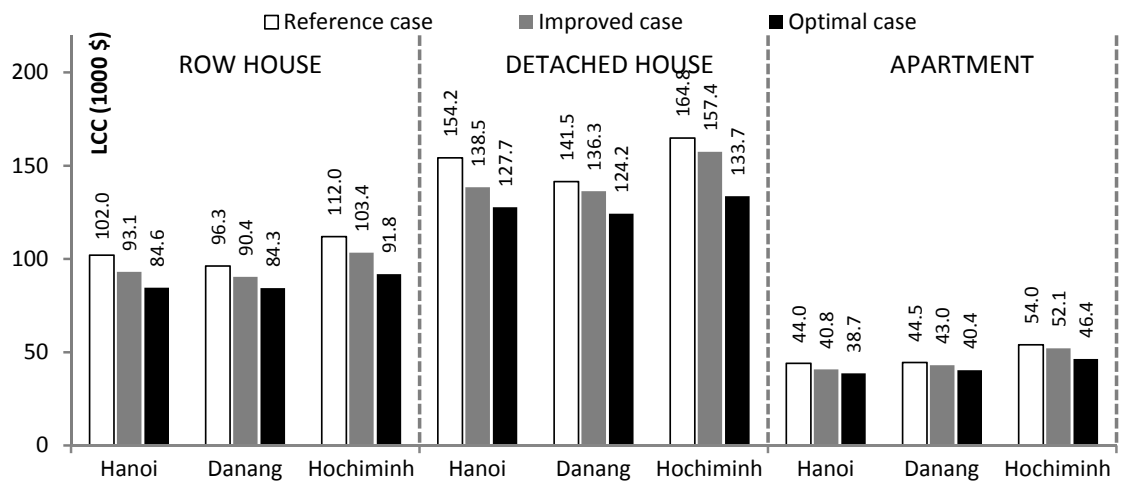

Figure 5: Comparison of the LCCs in the AC houses.

In improved NV houses, the TDHs were considerably reduced. The largest reduction of $70 \%$ was observed in the detached house under the climate of Danang. The smallest reduction was $19 \%$, observed in the apartment in Hochiminh city. In average, a $44.9 \%$ reduction of the THD was obtained. Using the PSM, the TDHs of $5 / 9$ cases was classified as "GOOD" (TDH $\leq 10 \%$ with mean exceeding temperature smaller than $1^{\circ} \mathrm{C}$ ) [5]. 
In improved AC houses, the LCCs were not reduced significantly as expected from the improvement in NV houses. The average reduction of LCCs was only $6.1 \%$.

\section{Efficiency of the SOM}

The PSM is usually time-consuming while it might yield only partial improvements because of the complex non-linear interactions of input variables on simulated results. To achieve an optimal (or near optimal) solution to a problem with less time and labor, the design target of a numerical building model is usually "solved" by iterative methods on computers, which construct infinite sequences, of progressively better approximations to a "solution", often known as the numerical optimization or the simulation-based optimization method (SOM).

This study used a two-step optimization method according to which the optimization procedure will be conducted through two phases, namely (i) sensitivity analysis and (ii) optimization.

\section{Sensitive analysis (SA)}

The philosophy of SA is that if we understand the relationships and the relative importance of design parameters on the building performance, we can easily improve the building performance by selecting appropriate design parameters. This study used the SA to eliminated unimportant (or low influential) design variables from the optimization. This helps to limit the number of optimization variables within an acceptable range (e.g. on the order of 10 [6]) associated with the solving capability of optimization algorithms.

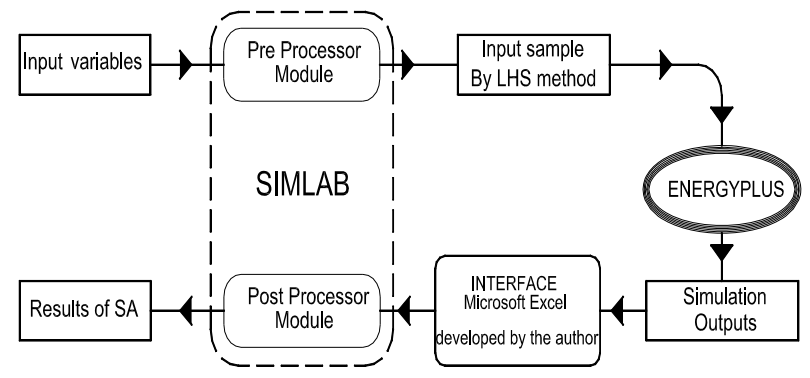

Figure 6: The full process of a SA using SimLab and EnergyPlus applied to this study.

This study used a Monte Carlo-based technique to perform the SA. Fig. 6 presents the procedure of SA applied to this study that employed SimLab (Available at http://ipsc.jrc.ec.europa.eu [Accessed 10/4/2013]) and EnergyPlus. The Standardized rank regression coefficient (SRRC) was used to measure the sensitivity of a variable. The SA was performed on both the NV and AC houses. 
Highly influential design variables were included in the optimization phase. More details of the SA and selected variables can be found in [8]. Fig. 7 shows an example of the SA result of the NV apartment.

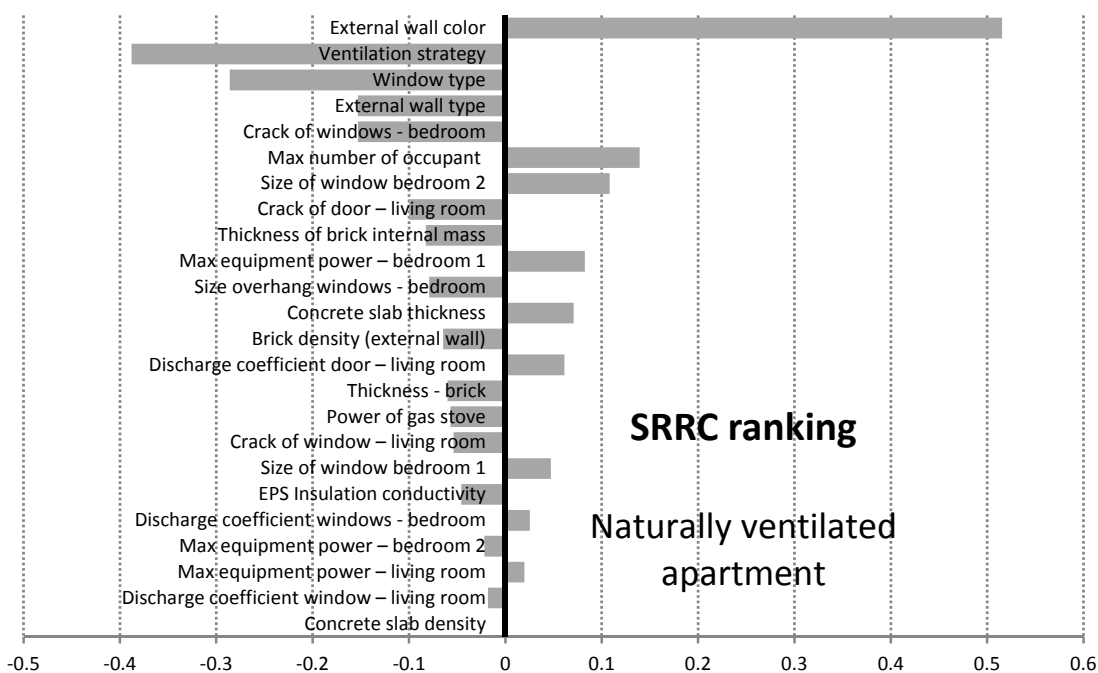

Figure 7: Variable sensitivity ranking using the SRRC in NV apartment under Danang climate.

\section{Optimization}

The optimization was done by coupling EnergyPlus and GenOpt [6] - a generic optimization tool. The optimization algorithm used was the hybrid form of the Particle swarm optimization and Generalized pattern search Hooke-Jeeves algorithm. This hybrid algorithm is capable to work efficiently on detailed building simulation problems where simulation outputs are generally non-linear, multi-model and highly discontinuous [9]. Optimization variables and settings of the optimization algorithm can be found in reference [8]. Objective functions to be minimized are the TDH and the LCC, applied to the NV and AC houses, respectively. The optimization was also performed on these housing models under 3 climates.

Figs 4 and 5 compared the TDHs / LCCs in optimal houses given by the SOM and the reference counterparts. In NV cases, it can be observed that the optimal cases offered great reductions of the TDH. The largest reduction of $99.9 \%$ occurred in the detached house in Hochiminh city. The smallest reduction of $43.7 \%$ can be observed in the detached house in Hanoi. The optimal cases always outperform the improved cases. Average TDH reduction given by the SOM was $86.1 \%$ while that of the PSM was only $44.9 \%$. It is worthy of note that seven of the optimal houses were nearly perfect, in term of the TDH. 
In $\mathrm{AC}$ cases, it can be observed that the LCC reductions were not as significant as the TDH reductions. The reduction amounts by the optimization were fairly uniform with an average reduction of $14.6 \%$ of the LCC.

\section{Discussion}

Firstly, this work considers the results from some studies in cold and temperate climate. In [10], the authors found that a reduction of $23-49 \%$ in the space heating energy for the optimized house could be achieved compared with the reference detached house. Most optimal solutions could be seen as Finnish low-energy houses. Similar to these results, Suh et al. [11] found 24\% and 33\% reduction of heating and cooling energy in a post office building in Korea using a knowledgebased design method and the SOM, respectively. By performing optimization on EnergyPlus models of an office under 3 climates of the U.S., Wetter and Wright [12] found a saving of an order of $7 \%$ to $32 \%$ of primary energy consumption, depending on the building location. In [13], optimal settings of optimization algorithms led to a reduction of $20.2 \%$ to $29.6 \%$ of the primary energy use by a large office building in temperate climates of the U.S. From these results, it is very likely that a reduction of $20 \%-30 \%$ of building energy consumption is an achievable target using the SOM.

However, the situation of warmer climates is likely not the same. In a study related to a large office building in the warm climate of Florida, U.S., Kampf et al. [13] found a reduction of total energy consumption of $7.1 \%$. This result shares the same trend found by this study in which the average reduction of LCCs was only $14.6 \%$.

Cost reduction in optimization of high-performance buildings seems to be very minor. Salminen et al. [14] tried to improve energy consumption of a LEEDcertified commercial building in Finland. They found that the optimization method could further reduce up to $10 \%$ of the annual energy consumption, accompanied by an additional investment of about 0.6 million Euros. Without the additional investment, improvement could only reach $1.1 \%$.

Cost reductions by the SOM clearly depend on the objective function to be minimized and many other factors (climates, building models, optimization algorithms...). Due to very limited results from the literature and the variety of the design objectives in optimization studies, a robust quantification of optimization efficiency needs further investigations.

\section{Conclusion}

This study reports a comprehensive framework developed to quantify the efficiency of the PSM and SOM in improving building performance. The performances of the reference cases (base cases) were carefully defined by the calibrated EnergyPlus models. The efficiencies of the PSM and SOM were obtained by applying these methods to 3 housing models, under 3 climate patterns in the tropic. 
This study found that the SOM is almost 2 times more efficient than the PSM in improving thermal comfort in NV houses and life cycle cost in AC houses. In average, the TDH could be reduced by $44.9 \%$ by the PSM while as high as $86.1 \%$ by the SOM, compared with the reference cases. The LCC had smaller reductions with $6.2 \%$ and $14.6 \%$ cut-off by the PSM and SOM, respectively.

However, it is important to stress that the efficiency of the PSM and SOM still depends on many other aspects of the study, especially the design objective to be improved. Results from other studies found in the literature also indicate that the cost reduction may also depend on the climate where cold climates tend to offer greater chances to reduce building energy cost.

By applying the SOM in design of NV buildings, the TDH of optimal solutions can be minimized for six of the nine case studies to nearly zero, revealing a significant potential of energy saving and the great feasibility of "zero energy buildings" concept in warm climates.

\section{Acknowledgements}

This research was financially supported by the Ministry of Education and Training of Vietnam and partly by Wallonie Bruxelles International.

\section{References}

[1] Nguyen, A.T. \& Reiter, S., An investigation on thermal performance of a low cost apartment in hot humid climate of Danang. Energy and Buildings, 47, pp. 237-246, 2012.

[2] Nguyen, A.T., Singh, M.K. \& Reiter, S., An adaptive thermal comfort model for hot humid South-East Asia. Building and Environment, 56, pp. 291-300, 2012.

[3] Nguyen, A.T. \& Reiter, S., Passive designs and strategies for low-cost housing using simulation-based optimization and different thermal comfort criteria. Journal of Building Performance Simulation, 7(1), pp. 68-81, 2014.

[4] Nguyen, A.T. \& Reiter, S., Optimum design of low-cost housing in developing countries using nonsmooth simulation-based optimization. Proceedings of the 28th International PLEA Conference, Lima, Peru, November 7-9, 2012.

[5] van der Linden, A.C., Boerstra, A.C. \& Kurvers, S.R., Thermal indoor climate as a building performance - Proposal for new criteria, design aids and assessment methods in The Netherlands. Proceedings of the International Conference on Advances in Building Technology, Hong Kong, China, December 4-6, 2002.

[6] Wetter, M., GenOpt, Generic optimization program - User manual, version 3.0.0. Technical report LBNL-5419, Lawrence Berkeley National Laboratory, 2009.

[7] Joint Research Centre - European Commission, Simlab 2.2 Reference Manual, Brussels: JRC, 2008. 
[8] Nguyen, A.T., Sustainable housing in Vietnam: climate responsive design strategies to optimize thermal comfort, $\mathrm{PhD}$ thesis: Université de Liège, 2013.

[9] Wetter, M. \& Wright, J.A., A comparison of deterministic and probabilistic optimization algorithms for nonsmooth simulation-based optimization. Building and Environment, 39, pp. 989 - 999, 2004.

[10] A. Hasan, A., Vuolle, M. \& Sirén, M., Minimisation of life cycle cost of a detached house using combined simulation and optimisation. Building and Environment, 43(12), pp. 2022-2034, 2008.

[11] Suh, W.J., Park, C.S. \& Kim, D.W., Heuristic vs. meta-heuristic optimization for energy performance of a post office building. Proceedings of the 12th Conference of International Building Performance Simulation Association, Sydney, Australia, November 14-16, 2011.

[12] Wetter, M. \& Wright, J.A., Comparison of a generalized pattern search and a genetic algorithm optimization method. Proceedings of the 8th IBPSA Conference, Eindhoven, Netherland, August 11-14, 2003.

[13] Kampf, J., Wetter, M. \& Robinson, D., A comparison of global optimisation algorithms with standard benchmark functions and real-world applications using EnergyPlus. Journal of Building Performance Simulation, 3, pp. 103$120,2010$.

[14] Salminen, M., Palonen, M. \& Sirén, K., Combined energy simulation and multi-criteria optimisation of a LEED-certified building. Proceedings of the building simulation and optimization conference, Loughborough, UK, September 10-11, 2012. 